\title{
Die sonografische Diagnostik bei Läsion des N. auricularis magnus
}

\begin{abstract}
WARUM DIESE FÄLLE?
Obwohl die elektrophysiologische

Technik zur Untersuchung des

Nervus auricularis magnus mit

Normwerten bereits länger

beschrieben ist $[1,2]$, sind die

Erfahrungen mit pathologischen

Fällen selten und auf einzelne

Kasuistiken beschränkt [3]. Alterna-

tiv kann dieser Nerv mittels

hochauflösender Sonografie nicht nur dargestellt werden. Bei verschiedenen Pathologien (idiopathisch, traumatisch, tumorös, iatrogen) ist es anschließend sogar möglich, nach Sicherung der Diagnose mit dem sonografiegesteuerten selektiven Block in einer Sitzung auch therapeutisch tätig zu werden [4]. Die beiden folgenden Kasuistiken beschreiben die gleichen morphologischen Veränderungen bei unterschiedlichen Schädigungsmechanismen (Punktionsverletzung und Druckschädigung) und zeigen, dass diese Methode zur Diagnosesicherung geeignet ist.
\end{abstract}

\section{Einleitung}

Eine Schädigung des Nervus auricularis magnus kann sich z. B. als Folge von Operationen im Gesicht und am Hals, Tumoren, langanhaltender Druckschädigung sowie selten idiopathisch mit der Klinik einer Neuralgie manifestieren [5]. Sensible Störungen im Versorgungsgebiet des Plexus cervicalis, meist durch Läsionen des N. cervicalis transversus und Nervus auricularis magnus, wurden bei $26 \%$ der Patienten nach einer Carotisdesobliteration diagnostiziert [6]. Der Nerv ist auch im Rahmen von kosmetischen Operationen (Facelifting, Narbenkorrektur) aufgrund seiner oberflächlichen Lage und des anatomischen Verlaufs auf der Lamina superficialis der Fascia cervicalis zur Ohrmuschel gefährdet. Der Nervus auricularis magnus entspringt aus den vorderen Ästen des zweiten und dritten Spinalnervs und erscheint zusammen mit anderen zervikalen Nerven am Hinterrand des Musculus sternocleidomastoideus. Der Nerv liegt in unmittelbarer Nähe an der Rückseite der Vena jugularis externa, verzweigt sich später meist in einen vorderen und einen hinteren Ast und zieht subkutan und vertikal-kreuzend in Verlaufsrichtung des Musculus sternocleidomastoideus bis zum Ohr [7].

Die folgenden Kasuistiken beschreiben eine Läsion des Nervs am Punctum nervorum im Rahmen einer Plexusanästhesie bzw.bei einer Druckschädigung durch Verbandmaterial.

\section{Fallberichte}

\section{Fall 1}

Der 72-jähriger Patient gab direkt nach einer zervikalen Plexusanästhesie eine Gefühlsstörung der Ohrmuschel und periaurikulär links ohne neuralgischen Schmerzen und Besserungstendenz an ( $\triangleright$ Abb. 1).
Die hochauflösende Sonografie mit einer $20 \mathrm{MHz}$-Sonde zeigte an der mit grün eingezeichneten Stelle am Hinterrand des Musculus sternocleidomastoideus eine segmentale hypoechogene Erweiterung des Nervus auricularis magnus im Querschnitt (Diameter 1,8, - Abb. 2). An dieser Stelle konnte ein positives Hoffmann-Tinel Zeichen ausgelöst werden. Im weiteren Verlauf nach kranial über den Musculus sternocleidomastoideus war der Diameter des Nervs

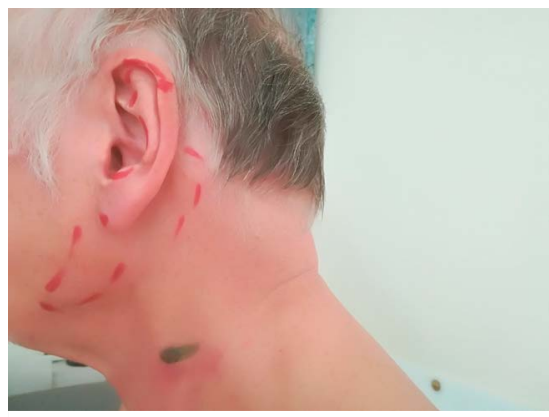

- Abb. 1 Grün eingezeichnet die Stelle der Nervenläsion am Hinterrand des Musculus sternocleidomastoideus am Punctum nervorum. Rot eingezeichnet die Ausdehnung der sensiblen Störung.

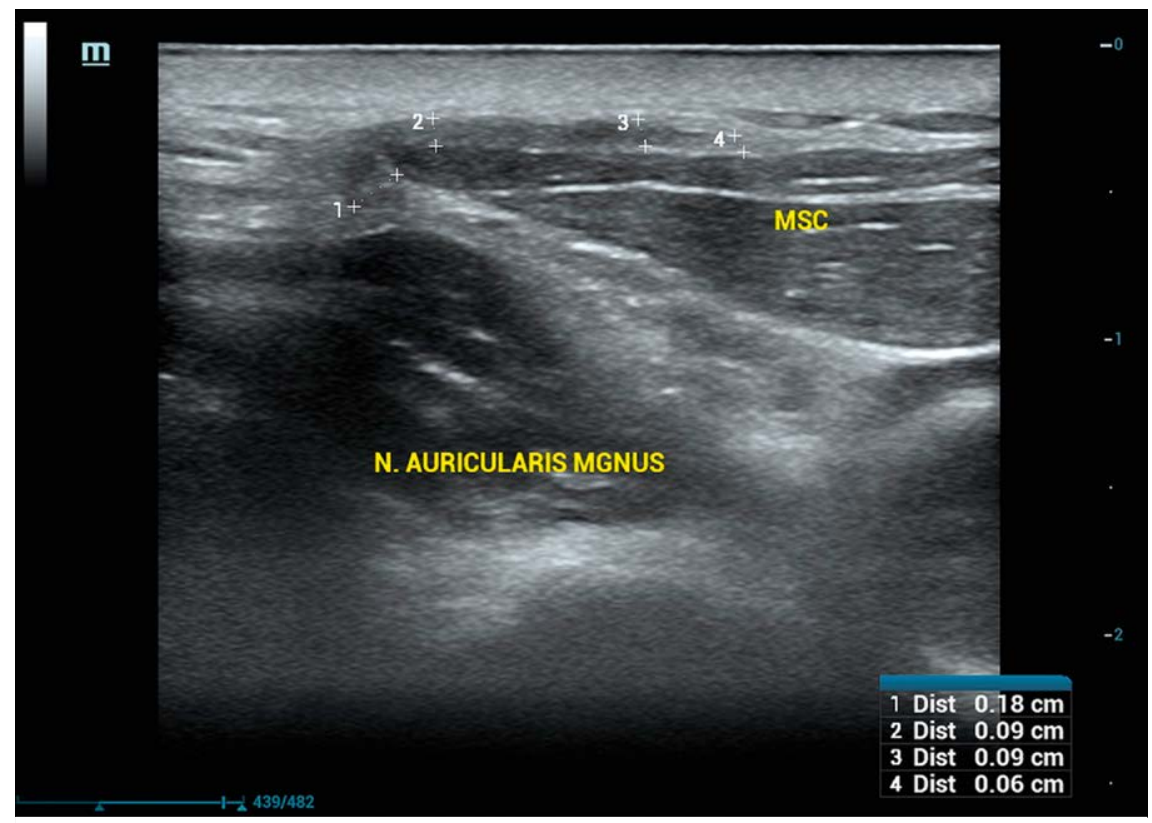

- Abb. 2 Hypoechogene Erweiterung des Nervus auricularis magnus in Höhe der Punktionsstelle am punctum nervorum (Diameter $1,8 \mathrm{~mm}$, mit 1 markiert). 


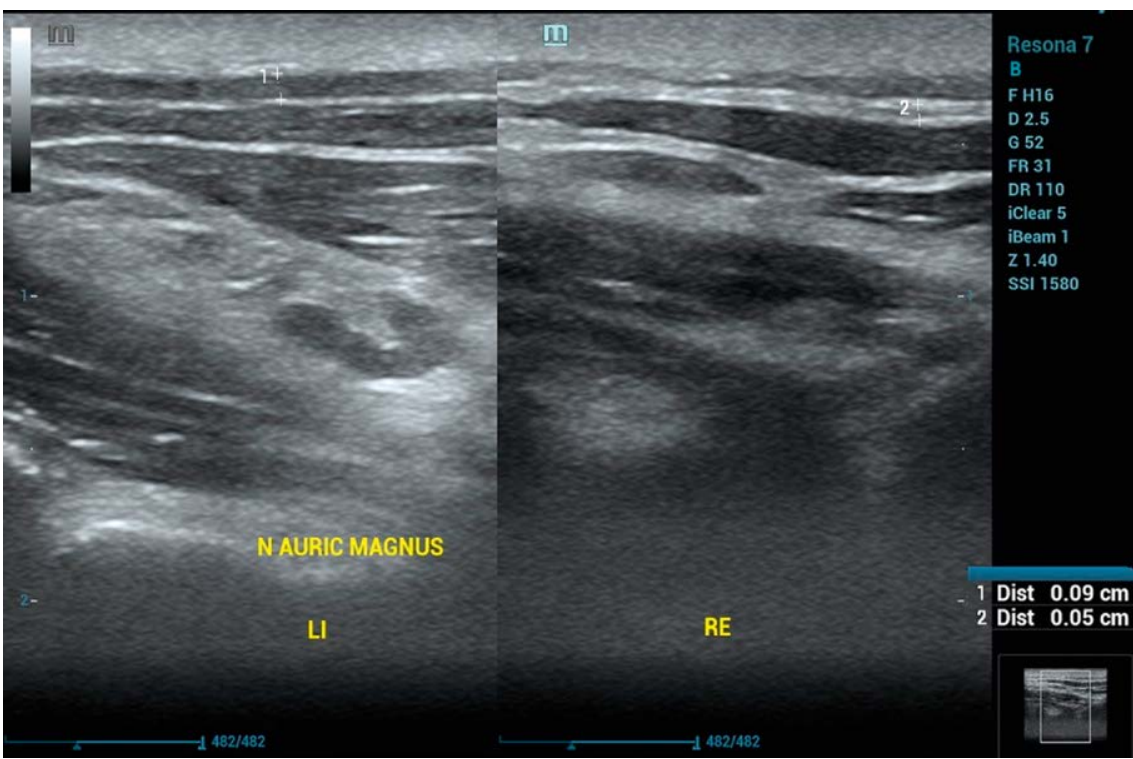

- Abb. 3 Diameter des Nervus auricularis magnus distal der Läsionsstelle auf der linken, betroffenen Seite mit $0,9 \mathrm{~mm}$ breiter als kontralateral auf der gesunden Seite $(0,5 \mathrm{~mm})$.

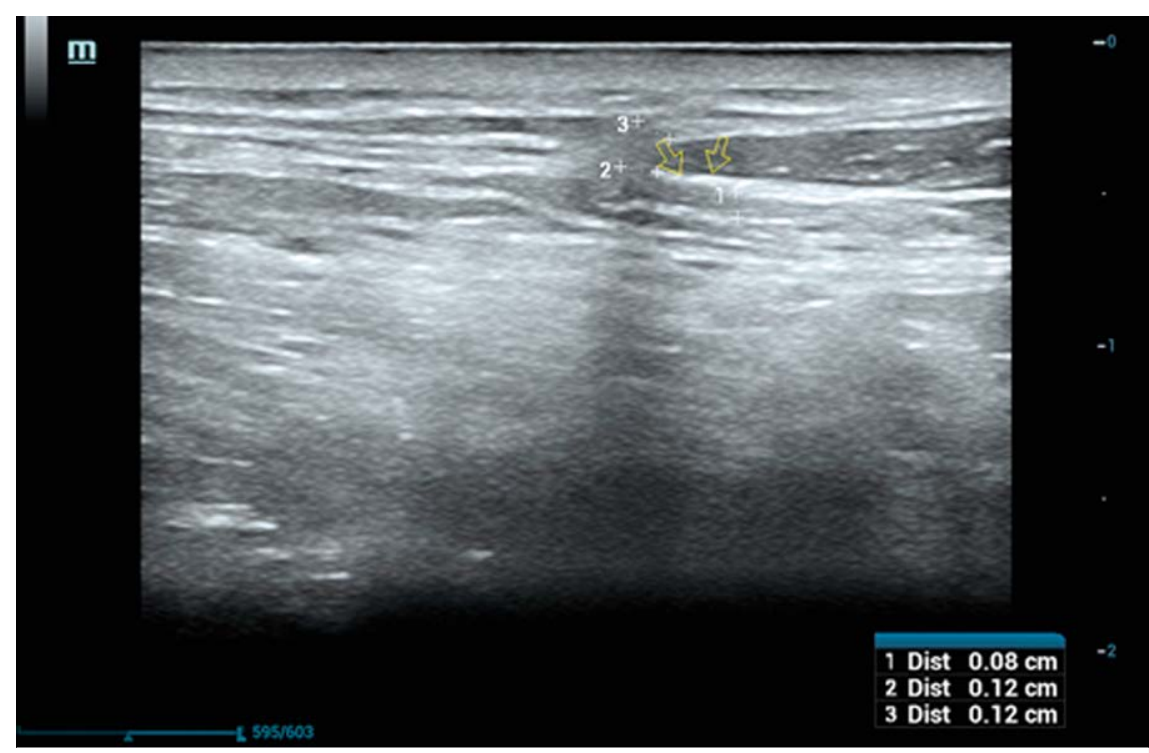

Abb. 5 Die kräftige hyperechogene Faszie des Musculus sternocleidomastoideus (Pfeil links) ist in direktem Kontakt zum erweiterten Nervus auricularis magnus (Pfeil rechts).

bei der longitudinalen Beschallung im Seitenvergleich erweitert (links $0,9 \mathrm{~mm}$, rechts $0,5 \mathrm{~mm}$, > Abb. 3).

\section{Fall 2}

Dem 68-jährigen Patienten war für die Zeit einer Schulteroperation ein Verband mit einem unteren straffen getapten Anteil angelegt, der quer über dem Musculus sternocleidomastoideus im mittleren Bereich verlief. Anschließend klagte er über eine Gefühlsstörung im Bereich der linken Ohrmuschel (॰ Abb. 4).

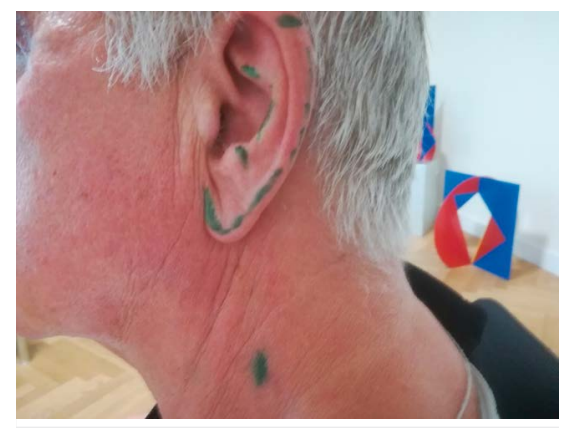

- Abb. 4 Ausdehnung des sensiblen Defizits und Läsionsort am Punctum nervorum.

\section{Diskussion}

Mithilfe der hochauflösenden Nervensonografie können auch Läsionen winziger kutaner Nerven nachgewiesen werden. In beiden Fällen trat die Schädigung des Nervus auricularis magnus am Punctum nervorum am hinteren Rand des M. sternocleidomastoideus auf. Beim ersten Fall trat die Läsion direkt nach einer Plexusanästhesie auf und die Beschwerden persistierten auch nach 2 Monaten, vermutlich infolge der direkten Schädigung des Nervs durch die Punktion. Beim zweiten Fall wurde der Nerv wohl durch einen straff angelegten Verband gegen die kräftige Faszie des Musculus sternocleidomastoideus gedrückt. In beiden Fällen wurde bei verschiedenen Schädigungsmechanismen die gleiche morphologische Veränderung, nämlich die hypoechogene Erweiterung des Nervs, nachgewiesen. Die hiesigen Normwerte auf der asymptomatischen Seite (Diameter $0,5 \mathrm{~mm}$ ) waren deutlich geringer als die in der zitierten [4] nervensonografischen Arbeit $(1,4 \mathrm{~mm} \pm 0,03)$. Bei meinen Kasuistiken wies lediglich die Erweiterung des Nervs um 0,3 mm im Seitenvergleich die Pathologie nach, der Absolutwert wäre allein nicht hilfreich gewesen. Wegen der nicht erklärlichen Diskrepanz bei den Normwerten sind weitere Untersuchungen erforderlich. Die sonografiegesteuerte Blockade dieses Nervs ist bereits eine bekannte Anwendung [4, 5] bei z. B. anästhesistisch und radiologisch-schmerztherapeutisch tätigen Ärzten, aber diese Methode könnte zukünftig auch zunehmend von Neurologen diagnostisch und therapeutisch angewandt werden. 
WAS LERNT MAN DARAUS?

Bei Läsionen des N. auricularis magnus kann die Sonografie durch den Nachweis einer ipsilateralen Erweiterung des Nervs diagnostisch und lokalisatorisch eingesetzt werden.

\section{Interessenkonflikt}

Die Autoren geben an, dass kein Interessenkonflikt besteht.

\section{Literatur}

[1] Palliyath SK. A technique for studying the greater auricular nerve conduction velocity. Muscle Nerve 1984; 7: 232-234

[2] Kimura I, Seki H, Sasao $S$ et al. The great auricular nerve conduction study: a technique, normative data and clinical usefulness. Electromyogr Clin Neurophysiol 1987; 27: 39-43

[3] Park SW, Bae JS, Jeong YS et al. Management of great auricular neuralgia confirmed by electrophysiological examination: A case report. Workshop of the Korean Academy of Rehabilitation Medicine 2018
[4] Lieba-Samal D, Pivec C, Platzgummer H et al. High-Resolution Ultrasound for Diagnostic Assessment of the Great Auricular Nerve-Normal and First Pathologic Findings. Ultraschall Med 2015; 36: 342-347

[5] Jeon TY, Kim S. Treatment of great auricular neuralgia with real-time ultrasound-guided great auricular nerve block: A case report and review of the literature. Medicine (Baltimore) 2017; 96: e6325

[6] Cnotliwy M, Gasińska M, Petriczko W et al. Permanent local nerve injuries after carotid endarterectomy. Wiad Lek 2005; 58: 375-378

[7] Schrank C, Levy Y, Die Neuralgie des N auricularis magnus (NAM) nach Face-NeckLift. J. f. Ästhet. Chirurgie 2009; 2: 37-40

Autor

Josef Böhm

Institute

Neurologische Privatpraxis, Berlin

\section{Korrespondenzadresse}

\section{Dr. Josef Böhm, PhD}

Neurologische Privatpraxis

Kurfürstendamm 69

10707 Berlin

info@neurologie-adenauerplatz.de

\section{Bibliografie}

DOI http://dx.doi.org/10.1055/a-0672-1754 Klin Neurophysiol 2018; 49: 233-235 (c) Georg Thieme Verlag KG Stuttgart . New York

ISSN 1434-0275 